\title{
Project management and scheduling
}

\author{
Erik Demeulemeester $\cdot$ Rainer Kolisch $\cdot$ Ahti Salo
}

Published online: 2 November 2012

(C) Springer Science+Business Media New York 2012

Project management and scheduling are major issues for every company or organization facing rapid changes in its environment. Furthermore, project scheduling techniques have become indispensable for the attainment of effectiveness and efficiency of processes. This special issue collects 11 carefully selected papers which deal with optimization or decision analysis problems in the field of project management and scheduling. It covers a considerable range of topics, including solution methods for classical project scheduling problems (Bianco and Caramia, Koné et al., Lim et al., Van Peteghem and Vanhoucke), mixed-integer programming (MIP) formulations in order to solve scheduling problems with commercial MIP-solvers (Bianco and Caramia, Koné et al.), models and solution algorithms for multi-project scheduling (Beşikci et al.), extensions of the classical resource-constrained project scheduling problem (Hartmann), models and solution approaches integrating decisions at different managerial levels such as selection and scheduling, or scheduling and control (Gutjahr and Froeschl, Hazir and Schmidt), risk in project management and scheduling (Tian and Demeulemeester, Artigues et al., Hazir and Schmidt, Gutjahr and Froeschl), and the assignment of resources to different stakeholders (Fink and Homberger, Beşikci et al.). The operations research techniques employed include common approaches such as metaheuristics (Lim et al., Fink and Homberger, Van Peteghem and Vanhoucke, Beşikci et al., Gutjahr

\footnotetext{
E. Demeulemeester

Research Center for Operations Management, KU Leuven, Leuven, Belgium

e-mail: erik.demeulemeester@kuleuven.be

R. Kolisch ( $($ )

TUM School of Management, Technische Universität München, Munich, Germany

e-mail: rainer.kolisch@wi.tum.de
}

\section{A. Salo}

Department of Mathematics and Systems Analysis, Aalto University, Helsinki, Finland e-mail: ahti.salo@aalto.fi 
and Froeschl) and mixed-integer programming (Bianco and Caramia, Koné et al.), but also specific approaches such as scenario-relaxation algorithms (Artigues et al.), a Lagrangian-based algorithm (Beşikci et al.), and a Frank-Wolfe type algorithm (Gutjahr and Froeschl). This special issue thus covers a broad range of models, algorithms and applications. It demonstrates that 55 years after the invention of CPM and PERT, the field is more innovative and lively than ever. We encourage the readers to seek inspiration from this unique collection of papers and to continue work in this area, for instance by applying these results in different application areas, by extending these models further, or by developing even better algorithms for solving them efficiently. In what follows, we give a brief summary of the papers.

\section{A new formulation for the project scheduling problem under limited resources}

Bianco and Caramia propose a new mathematical model for the resourceconstrained project scheduling problem to be solved by off-the-shelf commercial MIP solvers. The model employs three types of variables, two binary variables associated with the start time and the finish time of an activity, respectively, as well as a continuous variable associated with the amount of an activity in progress at a given time.

\section{Comparison of mixed integer linear programming models for the resource-constrained project scheduling problem with consumption and production of resources}

Koné, Artigues, Lopez and Mongeau extend three different mixed-integer programming models for the resource-constrained project scheduling problem to the more general case with cumulative resources. The models make it possible to apply standard off-the-shelf MIP-solvers. Computational results show the strength and weaknesses of the three models.

\section{New meta-heuristics for the resource-constrained project scheduling problem}

Lim, Ma, Rodrigues, Tan and Xiao develop two new metaheuristics for the resource-constrained project scheduling problem. The first of these builds on a simulated annealing framework which employs different neighborhoods as well as a forward-backward improvement scheme. The second builds on a genetic algorithm framework using two gene pools as well as the neighborhoods introduced for the simulated annealing framework. Experimental results show that both metaheuristics perform competitively and that the genetic algorithm is one of the best performing metaheuristics for the resource-constrained project scheduling problem. 


\section{Project scheduling with resource capacities and requests varying with time: a case study}

Hartmann presents an extension of the resource-constrained project scheduling problem where the resource demand of activities and the resource supply vary over time. As an application, he discusses a real-world medical research project. A multipass priority rule based heuristic with tournament selection is proposed as a solution procedure.

\section{An ant-based coordination mechanism for resource-constrained project scheduling with multiple agents and cash flow objectives}

Fink and Homberger consider the multi-agent variant of the resource-constrained project scheduling problem with discounted cash flow objective. In this problem, there are several independent project managers who are in charge of a subset of the activities of a single project. Each project manager wants to schedule his activities so that the discounted cash flow is maximized. The authors propose an ant-based solution procedure for the case that information on the activity network, activity durations as well as resource demand and resource availability is available to all project managers, but that information on the cash flow of activities is only available to the project manager in charge of these activities.

\section{An artificial immune system algorithm for the resource availability cost problem}

Van Peteghem and Vanhoucke tackle the resource availability cost problem where the activities of a single project have to be scheduled such that a project deadline is met and the sum of the weighted peak requirements of the resources is minimized. As a solution procedure, the authors propose a population-based metaheuristic.

\section{On the interaction between roadrunner and railway scheduling and priority lists or resource flow networks}

Tian and Demeulemeester consider the stochastic discrete time/resource trade-off problem where the stochastic work content of each activity can be processed in different modes. In an experimental study, they investigate the performance of activity priority lists or resource flow networks in combination with roadrunner or railway scheduling on different performance measures, including the expected project makespan. The results indicate that in smaller projects the optimal combination strongly depends on the selected performance measure, while in larger projects a combination of roadrunner scheduling with a resource flow network gives good results. 


\section{Robust optimization for resource-constrained project scheduling with uncertain activity durations}

Artigues, Leus and Nobibon present an extension of the resource-constrained project scheduling problem in which there is considerable uncertainty in the activity durations so that approaches employing distribution functions for activity durations cannot be readily applied. The authors apply concepts from robust discrete optimization and propose an optimal and a heuristic scenario-relaxation procedure.

\section{Resource dedication problem in a multi-project environment}

Beşikci, Bilge and Ulusoy consider the so-called resource dedication problem in which renewable and nonrenewable resources have to be exclusively assigned to the projects over the entire planning horizon and in which projects are scheduled with the objective of minimizing the total tardiness of all projects. The authors propose two promising solution procedures: a genetic algorithm and a Lagrangian relaxation based heuristic.

\section{An integrated scheduling and control model for multi-mode projects}

Hazir and Schmidt develop an integrated scheduling and control approach for the discrete time/resource trade-off problem. In this approach, the scheduling perspective aims at minimizing the project cost subject to a project deadline, whereas the control perspective serves to minimize the control effort for given activity durations. The authors propose a tabu search procedure for the scheduling problem and a constraint nonlinear programming approach for the control problem.

\section{Project portfolio selection under uncertainty with outsourcing opportunities}

Gutjahr and Froeschl consider the strategic stochastic project portfolio selection problem in which the projects' returns and resource requirements are uncertain. Apart from the selection of projects, it is necessary to decide how workers with different qualifications are assigned to projects over time. If the realized resource demand exceeds availability, the excess demand is met by acquiring external resources. The authors propose a two-step solution procedure in which the project selection problem is first solved with a variable neighborhood search algorithm, and then a Frank-Wolfe type algorithm is applied for the remaining staffing and scheduling problem. 


\section{Concluding remarks}

This special issue has greatly benefited from the cooperation among the authors, reviewers, and editors. We would like to express our sincere thanks to the reviewers for their excellent and timely refereeing. Last, but not least, we thank all authors for their contributions which made this special issue possible. 